\title{
A new tool for differentiating hepatocellular cancer cells: Patterned carbon nanotube arrays
}

\author{
Gokce Kucukayan-Dogu $^{\mathrm{a}, 1}$, Damla Gozen ${ }^{\mathrm{b}, 1}$, Verda Bitirim ${ }^{\mathrm{b}}$, Kamil Can Akcali ${ }^{\mathrm{c}}$, \\ Erman Bengu ${ }^{\mathrm{d}, *}$ \\ a Materials Science and Nanotechnology Graduate Program, Bilkent University, Turkey \\ ${ }^{\mathrm{b}}$ Department of Molecular Biology and Genetics, Bilkent University, Turkey \\ c Department of Biophysics, Faculty of Medicine, Ankara University, Turkey \\ d Department of Chemistry, Bilkent University, Turkey
}

\section{A R T I C L E I N F O}

\section{Article history:}

Received 15 February 2015

Received in revised form 6 May 2015

Accepted 9 May 2015

Available online 18 May 2015

\section{Keywords:}

Carbon nanotube

Cancer cell

Differentiation

Collagen

Patterning

Toxicity

\begin{abstract}
A B S T R A C T
We aimed to develop a new approach to detect the invasiveness and metastatic degree of hepatocellular carcinoma cells (HCC) based on their epithelial mesenchymal transition (EMT) status by using patterned carbon nanotubes (CNT) without any further surface functionalization. We used well differentiated HUH7 and poorly differentiated SNU182 cells to examine and compare their adhesive features on patterned CNTs. We found that the well differentiated HUH7 cells attached significantly more on the patterned CNTs than the poorly differentiated SNU182 cells due to the difference in epithelial and mesenchymal phenotypes of these cells. Collagen coated patterned CNTs having less roughness resulted in a decrease in the number of attached cells compared to non-coated patterned surfaces indicating that surface topography playing also a vital role on the cell attachment. LDH testing indicated no adverse, or thereof toxic effect of collagen coated or non-coated patterned surfaces on the HCC cells. The results of this study clearly suggest that patterned CNT surfaces can be used as a diagnostic tool to determine the invasiveness and metastatic level of HCCs. Hence, CNTs could be considered as a promising diagnostic tool for the detection of differentiation and invasiveness of the HCC cells.
\end{abstract}

(C) 2015 Elsevier B.V. All rights reserved.

\section{Introduction}

Cancer is a devastating disease and responsible for high mortality rates [1]. The aggressiveness of a cancer cell depends on its interaction with neighboring cellular structure, and the metastasis of these cells to distant organs is one of the main causes of cancer mortality $[2,3]$. Hence, the factors that regulate the attachment of cancer cells are one of the most heavily studied areas of biology. Epithelial-mesenchymal transition (EMT) is one of these processes that has important roles in the regulation of the adhesion of cancer cells leading to an increase in invasiveness and metastatic potentials [4].

During development, EMT is a normal physiological and required process and has critical roles in embryogenesis. Epithelial and mesenchymal cells are two different cell types with distinct cell morphologies and functions [4]. The cells with epithelial

\footnotetext{
* Corresponding author. Tel.: +90 3122902153; fax: +90 3122664068.

E-mail addresses: bengu@fen.bilkent.edu.tr, erbengu@gmail.com (E. Bengu).

1 Equal contributions of authors.
}

characteristic are polarized immobile cells which interact with basement membrane. On the other hand, cells in mesenchymal phenotype become more migratory and invasive with distinct morphology, protein expression and gene signatures. EMT is initiated by several steps including; activation of transcription factors such as Twist and Snail, expression of specific proteins and microRNAs, reorganization of cytoskeleton and production of certain enzymes as a result of which an epithelial cell gain mesenchymal characteristic $[5,6]$.

The cells undergoing EMT typically lose the expression of epithelial cell markers such as E-cadherin, and gain mesenchymal marker expressions, like $\alpha$-SMA, N-Cadherin, vimentin and fibronectin [6]. These cells lose their adhesive features to neighboring cells or to a surface or substrate such as extracellular matrix. Adhesion occurs from the action of family proteins, called cell adhesion molecules (CAMs) which cadherin is a member of this family. The tumors in early stages undergoing EMT process become aggressive malignancies with increased invasiveness, metastasis and survival abilities $[7,8]$. Cancer cells undergoing EMT lose their adhesive properties. Thus, the relation between the tumor aggressiveness and EMT was clearly shown in hepatocellular carcinoma 

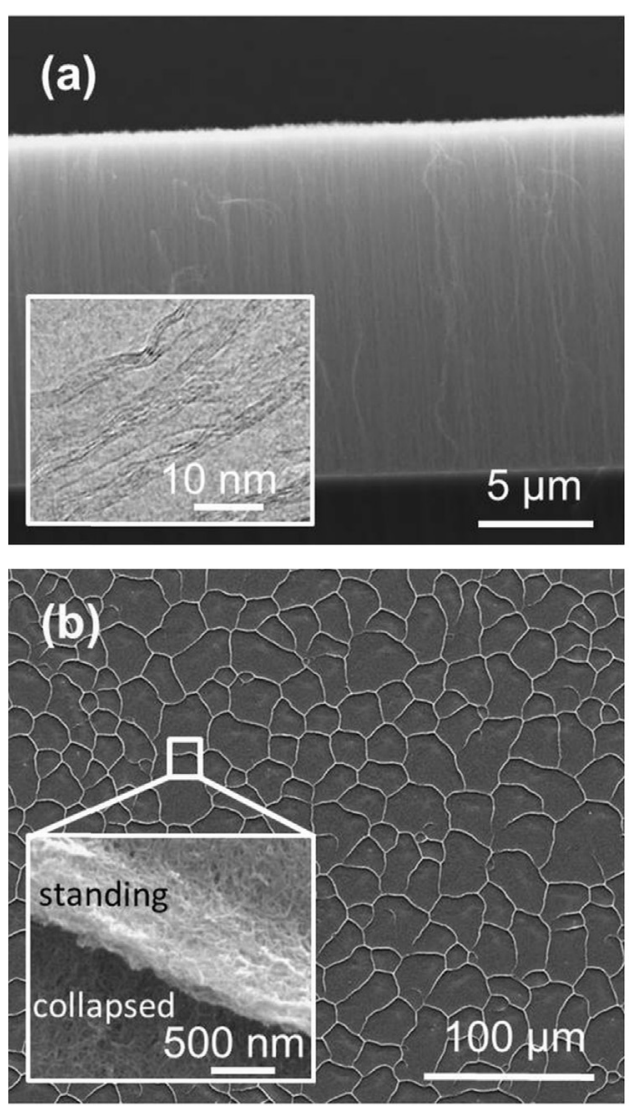

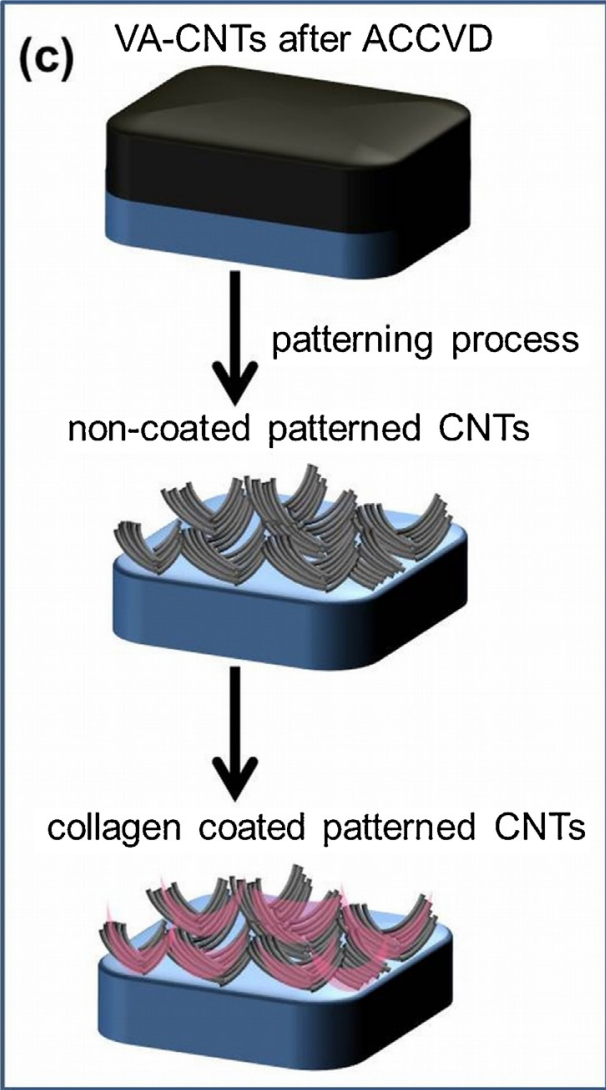

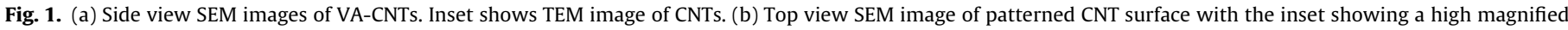
image from the wall of cavity. (c) Schematic representation of CNT surfaces after patterning and collagen coating.

(HCC) which is the third leading cause of world's cancer related mortality [9]. HCC is an epithelial cancer with four stages including well differentiated, moderately differentiated, poorly differentiated and undifferentiated tumors. The poorly differentiated cells gain mesenchymal phenotype through EMT to become more independent from the underlying tissue, with increased capacity to invade and metastase [10].

Novel strategies have been developed to classify and characterize the stages of cancer cells. The nanostructured materials such as nanowires [11], metal nanoparticles [12,13] and quantum dots [14] have been widely investigated both for cancer treatment and diagnosis applications. These recent advances in the field of biomaterials also triggered research on carbon nanotubes (CNTs) for the investigation of various cellular interactions [15]. There are number of studies reporting the use of CNTs as a nano-fibrous scaffolds for living cells [16-21]. Furthermore, the unique properties of CNTs prompted their application as a potential new tool for the detection of different types of cancer cells (oral, prostate and lung cancers) not only by taking advantage of their functionalization (e.g. binding specific markers on CNTs) [22-27] but also through exploiting their rigid surface properties for entrapping cancer cells $[28,29]$. For instance, improvement in the sensitivity and detection limits of cells have been achieved by the integration of CNTs in immunosensors [22,24,25].

In this study, we have employed patterned vertically aligned CNTs (VA-CNTs) as a tool to assess the invasiveness and metastatic degree of cancer cells based on their EMT properties. Our results showed that well differentiated HCC cells attached more on the patterned CNTs compared to the poor differentiated HCC cells due to their EMT characteristics without displaying any cytotoxic effects. Hence, our results suggest that VA-CNTs could be developed in to a promising tool for the detection of differentiation and invasiveness of the HCC cells.

\section{Materials}

\subsection{Synthesis and patterning of VA-CNTS}

VA-CNTs were grown by alcohol catalyzed chemical vapor deposition (ACCVD) method on oxidized Si (100) surfaces as described before in our previous study [18]. Sandwich catalyst layers $(\mathrm{Al} / \mathrm{Co} / \mathrm{Al})$ were prepared for the growth of VA-CNTs by using the electron beam and thermal evaporation techniques [18]. Si substrates with the aforementioned layers were introduced into the ACCVD furnace for the growth of VA-CNTs through reduction and reaction steps using ethanol as a carbon source at temperature of $625^{\circ} \mathrm{C}$ under flowing $\mathrm{H}_{2}$ and $\mathrm{Ar}$ gases $(20 \mathrm{sccm}$ and $100 \mathrm{sccm}$, respectively). After the growth, patterning was induced to the VACNTs by using a dropper filled with deionized water. Following this step, some of the patterned CNTs were treated with $1 \mu \mathrm{g} / \mu \mathrm{l}$ sterilized collagen solution for every $\mathrm{cm}^{2}$ (approximately 10:1 weight ratio of collagen to CNT) resulting in two separate groups of patterned CNT arrays; one non-coated and the other collagen coated.

\subsection{Cell culture}

We used two human HCC lines (SNU182 and HUH7) in our experiments. SNU182 is poorly differentiated and HUH7 is well differentiated hepatocellular cancer cell line. SNU182 cells were cultured in RPMI medium (Lonza, Verviers, Belgium) supplemented with $10 \%$ fetal bovine serum, $1 \%$ non-essential amino acids and 
1\% Penicillin/Streptomycin antibiotic. HUH7 cells were cultured in DMEM medium (Lonza) supplemented with $10 \%$ fetal bovine serum and $1 \%$ Penicillin/Streptomycin antibiotic.

\section{Methods}

\subsection{Total RNA isolation and reverse transcription}

HCC cells were trypsinized and total RNAs were extracted from the precipitate by using NucleoSpin RNA II Kit (MN MachereyNagel, Duren, Germany). ProtoScript M-MuLV First Strand cDNA Synthesis Kit (New England Biolabs, MA, USA) was used to prepare cDNAs from total RNA according to the manufacturer's protocol.

\section{2. $R T-P C R$}

All PCR reactions were performed with $1 \mu$ l cDNA, for 30 cycles. The amplifications for E-cadherin, Vimentin, Fibronectin, $\alpha$-SMA, N-Cadherin, Slug, Twist1, Sip1 and GAPDH were performed using Taq PCR Kit (New England Biolabs, MA, USA). The primers and amplicon sizes were listed in Table 1 . The initial denaturation step was performed at $94^{\circ} \mathrm{C}$ for $30 \mathrm{~s}$, followed by 30 cycles of denaturation at $94^{\circ} \mathrm{C}$ for $30 \mathrm{~s}$, annealing at $60^{\circ} \mathrm{C}$ for $30 \mathrm{~s}$ and extension at $68^{\circ} \mathrm{C}$ for $30 \mathrm{~s}$. The final extension step was performed at $68^{\circ} \mathrm{C}$ for $5 \mathrm{~min}$. The products were analyzed on $1.5 \%$ agarose gel.

\subsection{HCCS on patterned CNT surfaces}

The VA-CNTs were placed in 6 -well plates and $3 \times 10^{5}$ numbers of HUH7 and SNU182 cells were cultured on each well in $3 \mathrm{ml}$ medium. A negative control group with VA-CNT excluding any cultured cells was also prepared. At the 3rd day of cell culture, the cells were washed with PBS and prepared for imaging by scanning electron microscope (SEM) at low vacuum mode ( 40 bar) and accelerating voltage of $10 \mathrm{kV}$.

\subsection{Cytotoxicity of VA-CNTs surfaces on HCC}

The VA-CNTs were placed in 96-well plates in triplicates and $2 \times 10^{4}$ numbers of HUH7 and SNU182 cells were cultured on wells in $200 \mu \mathrm{l}$ medium. LDH Cytotoxicity Detection Kit (Clontech ${ }^{\circledR}$, Mountain View, CA) was used to measure the cytotoxicity according to the manufacturer's protocol. The readings were performed by using an ELISA reader at the wavelength value of $490 \mathrm{~nm}$. For the calculations, the average value of background group readings was subtracted from all of the sample readings.

\subsection{Statistical analysis}

All the results were analyzed with ANOVA. Minitab ${ }^{\circledR} 15$ Statistical Software was used to make multiple comparisons of control to experimental groups. All the comparisons were performed using Fisher's test with 95\% confidential interval. The graphs were drawn by the usage of GraphPad ${ }^{\circledR}$ program.

\section{Results and discussion}

\subsection{Preparation of VA-CNTS}

VA-CNTs were synthesized through ACCVD method on Si substrates using sandwich catalyst design $(\mathrm{Al} / \mathrm{Co} / \mathrm{Al})$ as we reported previously [18]. The side view SEM image of VA-CNTs revealed that CNTs were $10 \mu \mathrm{m}$ in height whereas their average diameters were around $10 \mathrm{~nm}$ shown by TEM analysis (Fig. 1a). Prior to the cell seeding step on CNTs, we created asperities for the

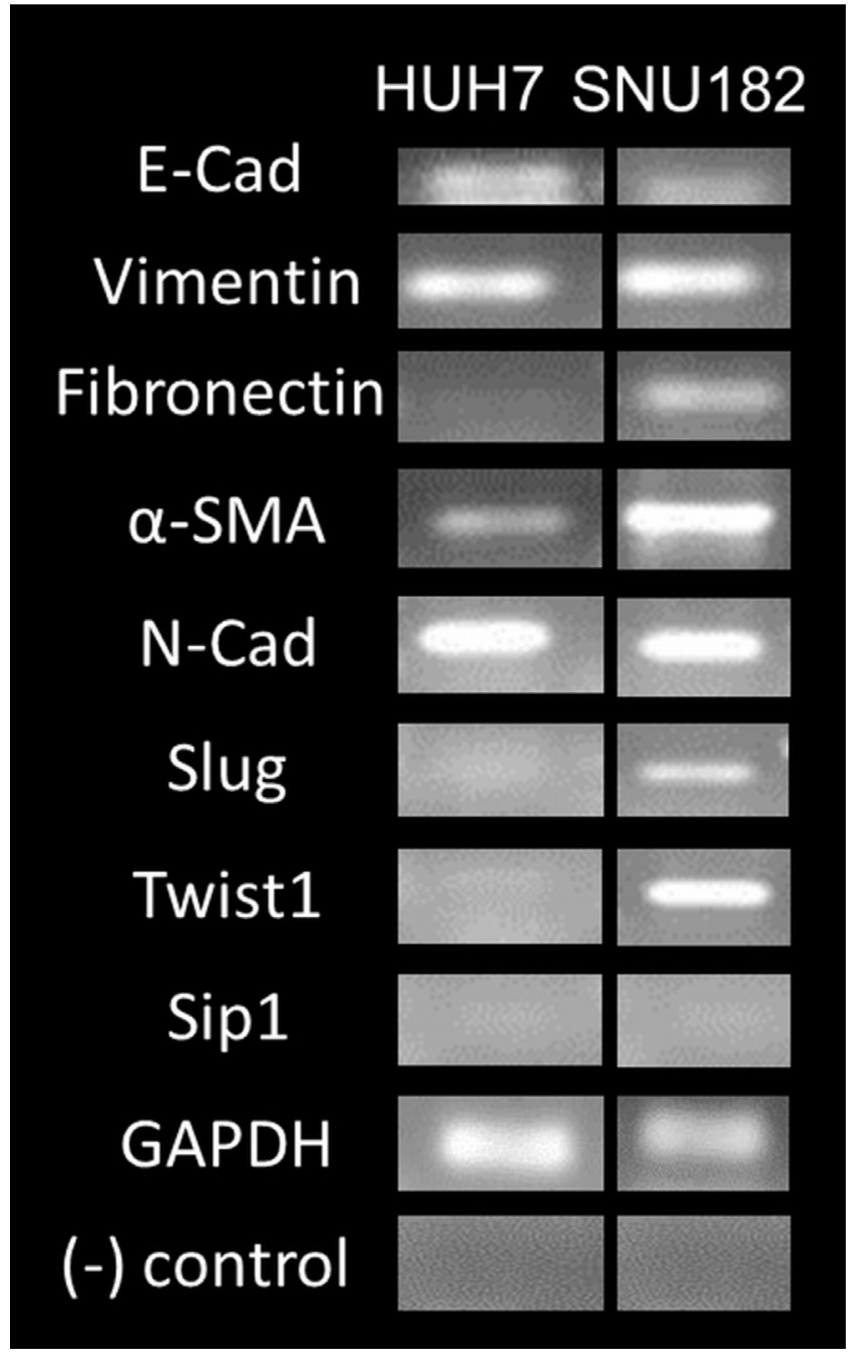

Fig. 2. EMT expressions of HUH7 and SNU182 cell lines at mRNA level.

growth. Aforementioned patterning step was induced by dropping deionized water on these surfaces. As a result of water contact of VA-CNTs, the aligned CNTs were collapsed and surrounded by standing CNTs forming cavities (Fig. 1b). The average width of these cavities was $10 \mu \mathrm{m}$. The mechanism behind the formation of selfassembled patterning is based on the elasto-capillary effect and hydrophobic property of aligned CNTs as explained by us and others previously $[18,30]$. Our experimental design of patterning was shown in Fig. 1c. Following the patterning process, patterned CNTs were either treated with collagen or not and were designated as patterned collagen coated and non-coated CNT surfaces thereafter.

\subsection{EMT marker expressions of HCC}

We monitored the EMT marker expression profiles of two HCC lines at mRNA level (Fig. 2). Our results showed that SNU182 cell line was positive for the expression of all the mesenchymal markers tested except Sip1 (Vimentin, Fibronectin, $\alpha$-SMA, N-Cad, Slug, Twist1). In contrary to SNU182, only three of the tested mesenchymal markers (Vimentin, $\alpha$-SMA and N-Cad.) were found to be expressed in HUH7 cells. Hence, the expression profiles of these cell lines confirmed that SNU182 cells are poorly differentiated whereas HUH7 cells are well differentiated HCC cell line [10]. In parallel to previous reports, our results also indicated that HUH7 had a more epithelial characteristic compared to SNU182 cells [10]. 
Table 1

Primer sequences used in PCR amplifications.

\begin{tabular}{lll}
\hline Gene name & Forward primer $\left(5^{\prime}-3^{\prime}\right)$ & Reverse primer $\left(5^{\prime}-3^{\prime}\right)$ \\
\hline E-Cadherin & GACTCGTAACGACGTTGCAC & GGTCAGTATCAGCCGCTTTC \\
Vimentin & GCAGGAGGAGATGCTTCAGA & ATTCCACTTTGCTTCAAGG \\
Fibronectin & AATATCTCGGTGCCATTTGC & CAGTAGTGCCTTCGGGACTG \\
$\alpha$-SMA & TATCAGGGGGCACCACTATG & GCTGGAAGGTGGACAGAGAG \\
N-Cadherin & TCCAGACCCCAATCAATTAATATTAC & AAAATCACCATTAAGCCGAGTGA \\
Slug & CTTTTTCTTGCCCTCACTGC & AGCAGCCAGATTCCTCATGT \\
Twist1 & GGAGTCCGCAGTCTTACGAG & TCTGGAGGACCTGGTAGAGG \\
SIP1 & TGTAGATGGTCCAGAAGAAATGAA & TTGGCAAAGTATTCCTCAAAATCT \\
GAPDH & GGCTGAGAACGGGAAGCTTGTCAT & CAGCCTTCTCCATGGTGGTGAAGA \\
\hline
\end{tabular}

\subsection{Proliferation and attachment of HCCS on patterned CNTS}

After the CNT surfaces were modified and sterilized by UV for one hour, poor (SNU182) and well (HUH7) differentiated HCC cells were seeded on both non-coated and collagen coated patterned CNT surfaces. The cells were incubated for 3 days on the patterned CNT surfaces and low vacuum SEM imaging was performed to assess the relation between their differentiation levels and attachment ability both on collagen coated and non-coated patterned
CNT surfaces (Fig. 3). Fig. 3a and b show SEM images of attached HUH7 cells on collagen coated and non-coated patterned CNT surfaces, respectively. Furthermore, attached SNU182 cells were also observed both on collagen coated and non-coated patterned CNT surfaces (Fig. 3c and d, respectively). For comparison of the number of attached cells on patterned CNTs, we counted the cells under SEM and average areal cell density was calculated dividing the total cell number on the surface to total surface area. The density plot given in Fig. 3e indicates that the average cell number for well
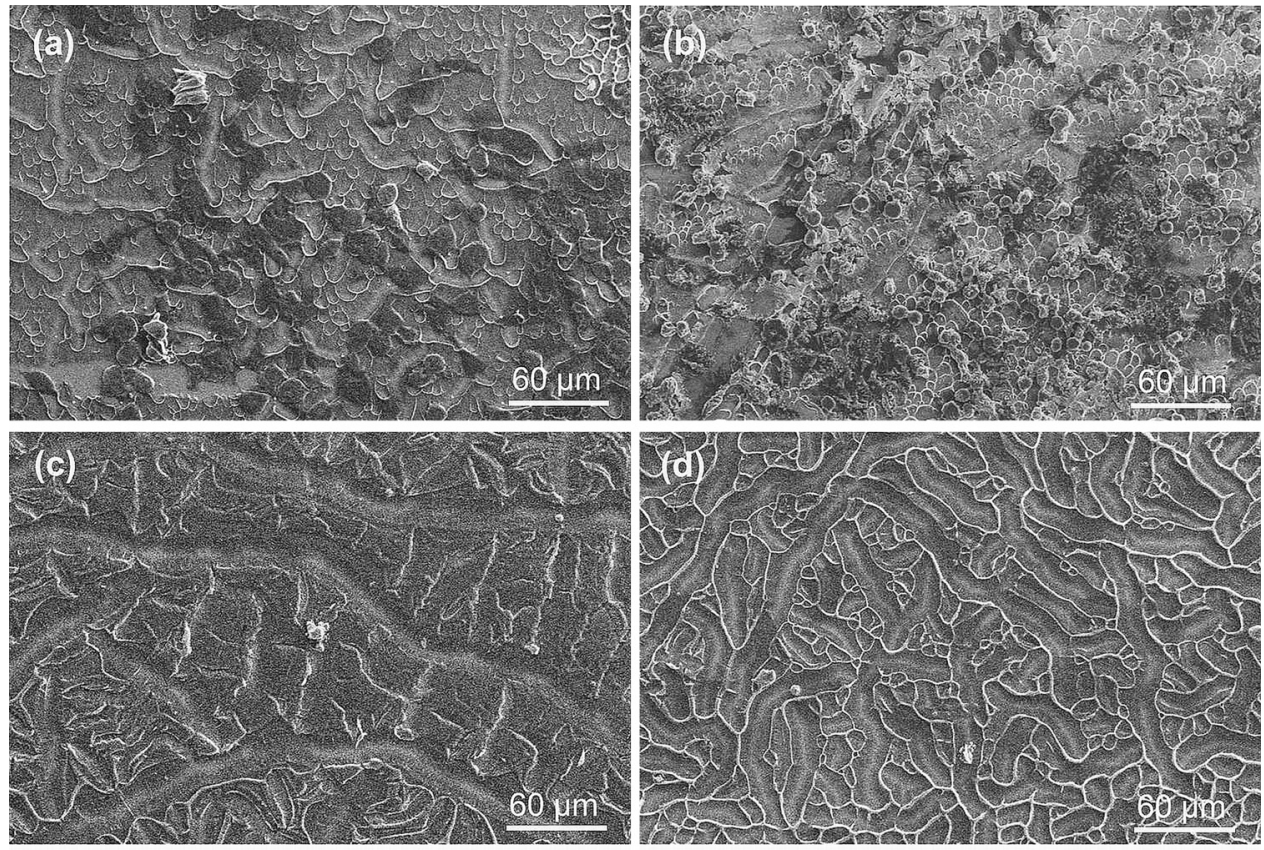

(e) $\quad \mathrm{HUH7}$

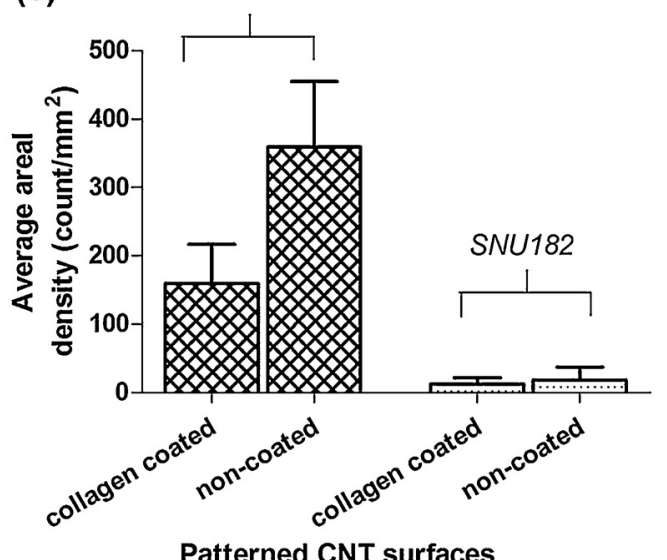

Patterned CNT surfaces

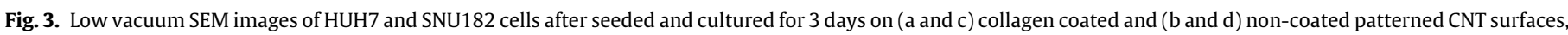
respectively. (e) Density plot for HUH7 and SNU182 cell number on collagen and non-coated patterned CNT surfaces. 


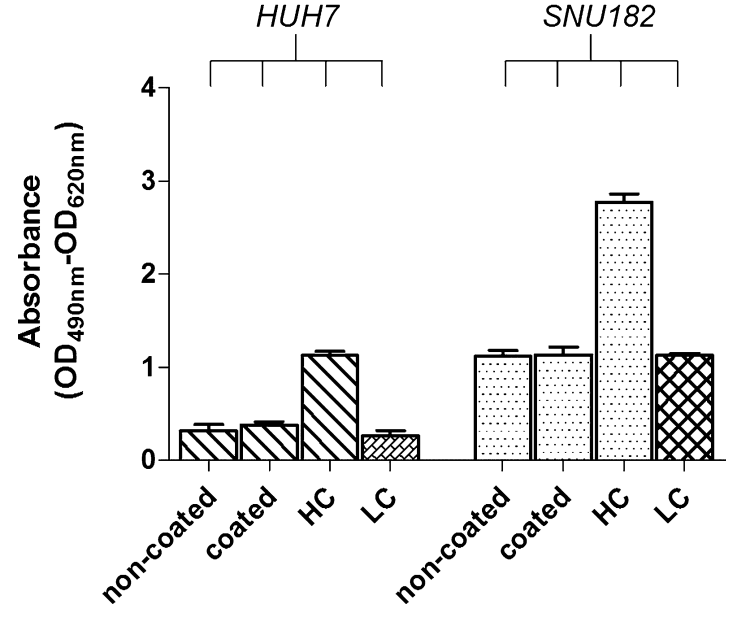

Patterned CNT surfaces

Fig. 4. LDH assay of cancer cells on non-coated and collagen coated CNT surfaces Low control (LC) and high control (HC) groups represent the case where there is no CNT surface.

differentiated cells were higher than the number for poor differentiated ones. The difference between the areal density of well and poor differentiated cells was statistically significant (significance value, $p<0.05$ ). This suggests that patterned CNT surfaces can be used for defining the differentiation level of HCC cells.

Collagen by itself has poor mechanical properties while CNTs have high elasticity and tensile strength in addition to their nanoscale dimensions [31,32]. Hence, to enhance the mechanical and functional properties of collagen, collagen blended with CNTs are used as composite biomaterials [33-35]. In our study, although collagen is used as a coating on the patterned CNTs, a significant effect of collagen is observed for the well differentiated cells. The number of well differentiated cells on collagen coated patterned CNTs was comparably lower than non-coated patterned CNTs. Contrarily, there was no significant effect of collagen coating on the attachment of poor differentiated cells which is likely reflecting the already low adhesive propensity of these cells (Fig. 3e).

It is well known that surface topography and roughness has a key role on the attachment of cells [36-38]. Collagen coating of CNTs may be smoothing the roughness by infiltrating and filling the voids between CNTs. Thereby, less surface area would be available for cell attachment which could be the reason for the observation of less number of cell attachment and growth for collagen coated surfaces. Several SEM images displaying the smoothing effect of the collagen coating are provided as supplementary information. The results summarized above suggest that the difference in the attachment ability of poor and well differentiated HCC cell lines on the non-coated CNT surfaces could be used for evaluating the differentiation level and thus aggressiveness of HCC cell lines.

Toxicity of CNTs has also been a controversial subject for researchers since there are conflicting reports on their toxicity $[17,39,40]$. Therefore, in order to investigate the cytotoxic effect of patterned CNT surfaces on HCC cell lines we performed LDH cytotoxicity analysis both on collagen coated and non-coated patterned CNTs (Fig. 4). In LDH test, two control groups were used; low control (LC) and high control (HC) groups in which HCC cells were seeded on bare plate surfaces. LC group shows the minimum LDH release while $\mathrm{HC}$ represents the maximum release. The closer the absorbance readings of the samples to $\mathrm{HC}$, the more cytotoxic the conditions are for that sample. Our LDH results clearly showed that HUH7 and SNU182 cells had minimum LDH release on the noncoated and coated patterned CNTs. This result indicates that CNTs are not cytotoxic for these HCC lines. This data also suggests that the difference in the attachment rates of the two cell lines are due to cells' interaction characteristics with CNT surfaces; not because of the cytotoxic effects of the CNT surfaces. Therefore, the well differentiated HCC line, HUH7 had greater tendency to attach on CNT surfaces when compared to the poor differentiated HCC line, SNU182.

The difference in the cancer cells ability to attach to patterned CNT arrays will be particularly an important tool for the diagnosis of the exact tumor cell type and differentiation status not only for hepatocellular cancer but for many different cancers. Molecular characterization of tumor cells is very important, since its characterization is the indicator of the classification of liver cancer similar to proposed for lung cancer $[41,42]$. Therefore, nanomedical device for the classification of hepatocellular cancer is important for the possible treatment of these tumors in the future.

\section{Conclusion}

We have demonstrated that patterned CNT surfaces have potential to distinguish between well differentiated HUH7 and poorly differentiated SNU182 cells. This is based on the difference in observed propensity of adhesion of HCCs on the collagen coated and non-coated patterned CNT surfaces according to their aggressiveness and metastatic grades. Our results showed that significantly more number of well differentiated HUH7 cells were observed on CNT surfaces than the poorly differentiated SNU182 cells irrespective of whether the CNT surfaces were coated with collagen or not $(p<0.05)$. However, this difference in the cell adhesion was not due to any toxic effect of CNTs, since the attached form of patterned CNTs on a rigid surface was found to have no toxic effects on the HCC cells as determined by LDH test. Furthermore, the number of attached cells was doubled on the non-coated patterned CNT surfaces when compared to collagen coated patterned surfaces, where collagen coating acted as a smoothing agent. Hence, it can be said that physical topography of the surfaces may have an important role on the HCC attachment. Through this technique metastatic grade of cancer cells could be detected in a fast and facile way, which could be used easily applied for diagnosis in order to determine the most effective therapy strategy depending on the tumor characteristics.

\section{Appendix A. Supplementary data}

Supplementary data associated with this article can be found, in the online version, at http://dx.doi.org/10.1016/j.apsusc.2015.05. 054

\section{References}

[1] A.A. Alghasham, Cucurbitacins - a promising target for cancer therapy, Int. J. Health Sci. 7 (2013) 77-89.

[2] L. Li, J. Cole, D.A. Margolin, Cancer stem cell and stromal microenvironment, Ochsner J. 13 (2013) 109-118.

[3] C.-B. Yeh, M.-J. Hsieh, C.-W. Lin, H.-L, Chiou, P.-Y. Lin, T.-Y. Chen, et al, The antimetastatic effects of resveratrol on hepatocellular carcinoma through the downregulation of a metastasis-associated protease by SP-1 modulation, PLOS ONE 8 (2013) 1-10.

[4] H. Acloque, M.S. Adams, K. Fishwick, M. Bronner-Fraser, M.A. Nieto, Epithelial-mesenchymal transitions: the importance of changing cell state in development and disease, J. Clin. Investig. 119 (2009) 1438-1449.

[5] R. Kalluri, R.A. Weinberg, The basics of epithelial-mesenchymal transition, J. Clin. Investig. 119 (2009) 1420-1428.

[6] J.P. Thiery, H. Acloque, R.Y.J. Huang, M.A. Nieto, Epithelial-mesenchymal transitions in development and disease, Cell 139 (2009) 871-890.

[7] T.-A. Liu, Y.-J. Jan, B.-S. Ko, S.-M. Liang, S.-C. Chen, J. Wang, et al., 14-3-3E Overexpression contributes to epithelial-mesenchymal transition of hepatocellular carcinoma, PLOS ONE 8 (2013) 1-12.

[8] M. Shiota, J.L. Bishop, K. Nip, A. Zardan, A. Takeushi, T. Cordonnier, et al., Hsp27 regulates epithelial mesenchymal transition, metastasis and circulating tumor cells in prostate cancer, Cancer Res. 73 (2013) 3109-3119. 
[9] C. Steinberg, S. Boudreau, F. Leveille, M. Lamothe, P. Chagnon, I. Boulais, Advanced hepatocellular carcinoma with subtotal occlusion of the inferior vena cava and a right atrial mass, Case Rep. Vasc. Med. 2013 (2013) 1-6.

[10] H. Yuzugullu, K. Benhaj, N. Ozturk, S. Senturk, E. Celik, A. Toylu, et al., Canonical Wnt signaling is antagonized by noncanonical Wnt5a in hepatocellular carcinoma cells, Mol. Cancer. 8 (2009) 1-20.

[11] C. Li, M. Curreli, H. Lin, B. Lei, F.N. Ishikawa, R. Datar, et al., Complementary detection of prostate-specific antigen using $\mathrm{In}_{2} \mathrm{O}_{3}$ nanowires and carbon nanotubes, J. Am. Chem. Soc. 127 (2005) 12484-12485.

[12] J. Zhang, B.P. Ting, M. Khan, M.C. Pearce, Y. Yang, Z. Gao, et al., Pt nanoparticle label-mediated deposition of Pt catalyst for ultrasensitive electrochemical immunosensors, Biosens. Bioelectron. 26 (2010) 418-423.

[13] Y. Zhuo, R. Yu, R. Yuan, Y. Chai, C. Hong, Enhancement of carcinoembryonic antibody immobilization on gold electrode modified by gold nanoparticles and $\mathrm{SiO}_{2}$ /thionine nanocomposite, J. Electroanal. Chem. 628 (2009) 90-96.

[14] M. Yang, A. Javadi, S. Gong, Sensitive electrochemical immunosensor for the detection of cancer biomarker using quantum dot functionalized graphene sheets as labels, Sens. Actuators B 155 (2011) 357-360.

[15] S. Ji, C. Liu, B. Zhang, F. Yang, J. Xu, J. Long, et al., Carbon nanotubes in cancer diagnosis and therapy, Biochim. Biophys. Acta 1806 (2010) 29-35.

[16] A.O. Lobo, E.F. Antunes, A.H.A. Machado, C. Pacheco-soares, Cell viability and adhesion on as grown multi-wall carbon nanotube films, Mater. Sci. Eng. C 28 (2008) 264-269.

[17] A.O. Lobo, M.A.F. Corat, E.F. Antunes, M.B.S. Palma, C. Pacheco-Soares, E.E. Garcia, et al., An evaluation of cell proliferation and adhesion on vertically-aligned multi-walled carbon nanotube films, Carbon 48 (2010) 245-254.

[18] V.C. Bitirim, G. Kucukayan-Dogu, E. Bengu, K.C. Akcali, Patterned carbon nanotubes as a new three-dimensional scaffold for mesenchymal stem cells, Mater. Sci. Eng. C 33 (2013) 3054-3060.

[19] M.A. Correa-Duarte, N. Wagner, C. Morsczeck, M. Thie, M. Giersig, Fabrication and biocompatibility of carbon nanotube-based 3D networks as scaffolds for cell seeding and growth, Nano Lett. 4 (2004) 2233-2236.

[20] S. Namgung, K.Y. Baik, J. Park, S. Hong, Controlling the growth and differentiation of human mesenchymal stem cells by the arrangement of individual carbon nanotubes, ACS Nano. 5 (2011) 7383-7390.

[21] X. Li, H. Liu, X. Niu, B. Yu, Y. Fan, Q. Feng, et al., The use of carbon nanotubes to induce osteogenic differentiation of human adipose-derived MSCs in vitro and ectopic bone formation in vivo, Biomaterials 33 (2012) 4818-4827.

[22] A. Salimi, B. Kavosi, F. Fathi, R. Hallaj, Highly sensitive immunosensing of prostate-specific antigen based on ionic liquid-carbon nanotubes modified electrode: application as cancer biomarker for prostatebiopsies, Biosens. Bioelectron. 42 (2013) 439-446.

[23] S. Chen, R. Yuan, Y. Chai, L. Min, W. Li, Y. Xu, Electrochemical sensing platform based on tris(2,2'-bipyridyl)cobalt(III) and multiwall carbon nanotubes-nafion composite for immunoassay of carcinoma antigen-125, Electrochim. Acta 54 (2009) 7242-7247.

[24] X. Yu, B. Munge, V. Patel, G. Jensen, A. Bhirde, J.D. Gong, et al., Carbon nanotube amplification strategies for highly sensitive immunodetection of cancer biomarkers, J. Am. Chem. Soc. 128 (2006) 11199-111205.

[25] R. Malhotra, V. Patel, J.P. Vaqué, J.S. Gutkind, J.F. Rusling, Ultrasensitive electrochemical immunosensor for oral cancer biomarker IL-6 using carbon nanotube forest electrodes and multilabel amplification, Anal. Chem. 82 (2010) 3118-3123.
[26] B.V. Chikkaveeraiah, A. Bhirde, R. Malhotra, V. Patel, J.S. Gutkind, J.F. Rusling Single-wall carbon nanotube forest arrays for immunoelectrochemical measurement of four protein biomarkers for prostate cancer, Anal. Chem. 81 (2009) 9129-9134.

[27] G. Peng, E. Trock, H. Haick, Detecting simulated patterns of lung cancer biomarkers by random network of single-walled carbon nanotubes coated with nonpolymeric organic materials, Nano Lett. 8 (2008) 3631-3635.

[28] M. Abdolahad, Z. Sanaee, M. Janmaleki, S. Mohajerzadeh, M. Abdollahi, M. Mehran, Vertically aligned multiwall-carbon nanotubes to preferentially entrap highly metastatic cancerous cells, Carbon 50 (2012) 2010-2017.

[29] M. Abdolahad, M. Taghinejad, H. Taghinejad, M. Janmaleki, S. Mohajerzadeh, A vertically aligned carbon nanotube-based impedance sensing biosensor for rapid and high sensitive detection of cancer cells, Lab. Chip. 12 (2012) 1183-1190.

[30] C.T. Wirth, S. Hofmann, J. Robertson, Surface properties of vertically aligned carbon nanotube arrays, Diam. Relat. Mater. 17 (2008) 1518-1524.

[31] J.N. Coleman, U. Khan, W.J. Blau, Y.K. Gun'ko, Small but strong: a review of the mechanical properties of carbon nanotube-polymer composites, Carbon 44 (2006) 1624-1652

[32] M.P.E. Wenger, L. Bozec, M.A. Horton, P. Mesquida, Mechanical properties of collagen fibrils, Biophys. J. 93 (2007) 1255-1263.

[33] T.D.B. Nguyen-vu, H. Chen, A.M. Cassell, R.J. Andrews, M. Meyyappan, Vertically aligned carbon nanofiber architecture as a multifunctional 3-D neural electrical interface, IEEE Trans. Biomed. Eng. 54 (2007) 1121-1128.

[34] P.R. Supronowicz, P.M. Ajayan, K.R. Ullmann, B.P. Arulanandam, D.W. Metzger, R. Bizios, Novel current-conducting composite substrates for exposing osteoblasts to alternating current stimulation, J. Biomed. Mater. Res. 59 (2002) 499-506.

[35] Y. Cho, R.B. Borgens, The effect of an electrically conductive carbon nanotube/collagen composite on neurite outgrowth of PC12 cells, J. Biomed. Mater. Res. 95 (2010) 510-517.

[36] S. Oh, K.S. Brammer, Y.S.J. Li, D. Teng, A.J. Engler, S. Chien, et al., Stem cell fate dictated solely by altered nanotube dimension, Proc. Natl. Acad. Sci. U. S. A. 106 (2009) 2130-2135.

[37] T.P. Kunzler, C. Huwiler, T. Drobek, J. Vörös, N.D. Spencer, Systematic study of osteoblast response to nanotopography by means of nanoparticle-density gradients, Biomaterials 28 (2007) 5000-5006.

[38] R.L. Price, K. Ellison, K.M. Haberstroh, T.J. Webster, Nanometer surface roughness increases select osteoblast adhesion on carbon nanofiber compacts, J. Biomed. Mater. Res. 70 (2004) 129-138.

[39] A.O. Lobo, M.A.F. Corat, E.F. Antunes, S.C. Ramos, C. Pacheco-Soares, E.J. Corat, Cytocompatibility studies of vertically-aligned multi-walled carbon nanotubes: Raw material and functionalized by oxygen plasma, Mater. Sci. Eng. C 32 (2012) 648-652.

[40] S.K. Smart, A.I. Cassady, G.Q. Lu, D.J. Martin, The biocompatibility of carbon nanotubes, Carbon 44 (2006) 1034-1047.

[41] O. Barash, N. Peled, F.R. Hirsch, H. Haick, Sniffing the unique odor print of non-small cell lung cancer with gold nanoparticles, Small 5 (2009) $2618-2624$.

[42] O. Barash, N. Peled, U. Tisch, P.A. Bunn, F.R. Hirsch, H. Haick, Classification of lung cancer histology by gold nanoparticle sensors, Nanomedicine 8 (2012) $580-589$. 